\title{
Un cardinale inquisito e i conflitti religiosi del Cinquecento
}

Fu Tommasino de' Bianchi, cronista della Modena del Cinquecento, a raccontare che il 30 maggio 1542, durante una solenne cerimonia nel duomo della città, il vescovo Giovanni Morone si era lasciato sfuggire di mano il venerato reliquiario contenente il braccio del santo patrono Geminiano, caduto a terra e rimasto „tutto storto et amachato“ (p. 111). Tra la folla dei fedeli l'incidente aveva suscitato vivo sconcerto, e i maligni vi avevano scorto un segno di malaugurio per una comunità il cui pastore appariva loro non del tutto ortodosso. Figura che divise in vita, quella di Morone avrebbe continuato a farlo dopo la morte: come si evince dal capitolo finale di questa esaustiva ricerca, se per un verso la storiografia teatina ne avrebbe tramandato un ritratto assai ostile, altri (dagli eredi di Cesare Baronio a Girolamo Tiraboschi, da Ferdinando Ughelli e Pietro Sforza Pallavicino a Hubert Jedin) ne avrebbero difeso la memoria di vescovo e di cardinale che avrebbe avuto il merito di chiudere un travagliato concilio e di favorire gli indirizzi della ripresa cattolica e tridentina (in modo più significativo, Paolo Sarpi si astenne dai giudizi, p. 884).

Il volume di Firpo e Maifreda sceglie il taglio biografico, che da anni conosce un revival, per offrire un solido affresco dei conflitti spirituali e politici dell'Italia e dell'Europa del Cinquecento. Programmaticamente antiquato nello stile e nella scelta di valorizzare l'erudizione e l'ancoraggio stringente a centinaia di fonti a stampa e manoscritte (con il gusto della citazione puntuta e brillante), il libro è una sfida al lettore - tanto vuole essere „torrenziale, onnivoro, scoraggiante“ (p. XXIX). Ma si tratta appunto di una sfida, che ripagherà ampiamente chi lo prenda in mano anche solo per ripercorrere una delle numerose pagine controverse della storia religiosa di un'epoca contrastata e cruciale. Perché se Firpo ha già avuto modo di raccontarci Morone attraverso saggi e monografie, e soprattutto grazie alla doppia edizione delle carte legate al processo inquisitoriale che fu imbastito contro di lui, ora la figura del cardinale può emergere a tutto tondo, a partire dal contesto in cui mosse i primi passi. Si allude a quel ducato di Milano che ci viene restituito con tonalità chabodiane e in cui ascese la famiglia del protagonista: anzitutto Girolamo, il padre, cancelliere dell'ultimo Sforza e, nello scorcio finale delle guerre d'Italia, uomo di fiducia degli Asburgo. Chierico „quasi per caso“ (p. 31), grazie ai legami politici del padre, ancora giovane Giovanni divenne vescovo di Modena, e in quella veste nunzio alla corte imperiale di Ferdi-

Recensione di: Massimo Firpo/Germano Maifreda, L'eretico che salvò la Chiesa. Il cardinale Giovanni Morone e le origini della Controriforma, Torino (Einaudi) 2019 (Einaudi Storia 84), XXXIII, 1122 pp., ill., ISBN 978-88-06-23357-0, € 48.

Kontakt: Vincenzo Lavenia, vincenzo.lavenia@unibo.it 
nando (1536). Fu da quel momento che Morone acquisì grande abilità diplomatica e consolidò un rapporto con il mondo germanico che dalle pagine del libro appare come un filo rosso della sua vicenda e che più tardi si sarebbe riflesso nelle posizioni curiali del porporato, nella scelta di promuovere la fondazione del Collegio Germanico, nei progetti di crociata che portarono allo scontro di Lepanto e nell'ovvia primazia che gli venne riconosciuta all'interno della Congregazione Germanica istituita nel 1573 (p. 798). Consapevole sin dal primo viaggio Oltralpe che per ricomporre la divisione dell'Impero la Chiesa di Roma dovesse scendere a compromessi e riformarsi rapidamente, Morone si avvicinò alla linea di Gasparo Contarini e fu in contrasto con quella di Girolamo Aleandro, il legato di Worms. Firpo e Maifreda narrano il naufragio delle proposte di compromesso teologico tra gli opposti fronti, fino alla dieta di Regensburg e oltre; e mettono a fuoco come gli indirizzi politico-religiosi di Morone si siano rispecchiati nel primo dei due momenti in cui l'uomo resse la diocesi di Modena.

Tra le eminenti figure elevate al cardinalato da Paolo III, Morone non poteva vantare il pedigree umanistico di un Pietro Bembo, ma la sua attenzione per le questioni dottrinali fu acuita dai dibattiti circa la materia più scottante di quegli anni: la giustificazione per fede. Così nei capitoli III e IV gli autori ripercorrono il noto tentativo intrapreso da Morone per arginare senza processi né clamori l',,infezione“ ereticale che dilagava a Modena, venendo incontro con sensibilità contariniana al dotto circolo riunito nell'Accademia di Giovanni Grillenzoni. E connettono quanto accadeva in Germania con gli scontri che si aprirono in Curia dal momento in cui fu istituito il Sant'Uffizio e Morone fu inviato a Trento quale legato pontificio destinato a guidare un concilio rinviato prima da Clemente VII e poi dall'anziano papa Farnese (1542). Fu in quell'occasione che il politico Morone fu „,convertito“ al valdesianesimo da Marcantonio Flaminio e da Reginald Pole, punto di riferimento degli „spirituali“: un breve sbandamento, se così può dirsi, che gli sarebbe costato l'implacabile persecuzione prima di Giampietro Carafa e poi di Michele Ghislieri. Ai loro occhi - e agli occhi di tutto il fronte intransigente che si coagulò intorno al Sacro Tribunale contro ogni ipotesi di scendere a compromessi aiutando così la strategia asburgica - le relazioni allora intrattenute da Morone ne avrebbero fatto un „eretico“ per sempre, sia pure abile a dissimularsi. Né sfuggì a chi aveva in mente di liquidare ogni apertura la linea secondo cui Morone operò negli anni in cui fu legato a Bologna e vescovo di Modena, prima di trasferire la diocesi al frate Egidio Foscarari, intessendo rapporti pericolosi e lasciando libero corso a predicatori eterodossi quando Bernardino Ochino già aveva varcato le Alpi.

La stagione del Morone „spirituale“ si sarebbe consumata tra il 1545 - anno di apertura del concilio - e il 1549, quando alla morte di Paolo III - per cui Morone aveva trattato gli intrighi di Piacenza, schivando una personale accusa di congiura anti-farnesiana - Pole fu liquidato come candidato al trono di Pietro da un Carafa che in conclave sventolò le carte che avrebbero provato l'eresia del cardinale anglico e angelico (l'esistenza di un processo contro di lui fu tuttavia un bluff, p. 321). Il partito imperiale aveva perduto, incapace come fu di elaborare una linea efficace contra- 
ria a quella intransigente, e un debole Giulio III cercò di arginare il partito di Carafa senza tuttavia limitare il potere che il Sant'Uffizio si conquistò sul campo. Pole prima di morire andava in Inghilterra a restaurare l'obbedienza romana a fianco della sanguinaria Maria; Pier Paolo Vergerio smascherava da fuoriuscito le ambiguità degli „spirituali“; Morone si recava nella diocesi di Novara e lì avviava la repressione del dissenso, entrando in relazione con un predicatore inquieto e per lui pericoloso come Lorenzo Davidico. Vescovo che si sforzava di somigliare a un „arcigno“ giudice della fede (p. 365), a Roma Morone divenne comunque il primo e ossessivo bersaglio delle inchieste di Carafa sino alla svolta consumatasi nel 1555.

Tra i meriti del libro c'è quello di restituirci le divisioni all'interno dell'Ordine domenicano e la complessità di figure come Girolamo Federici (poi nunzio presso i Savoia) e Marcello Cervini, che giocò una partita a sé, progressivamente vicina a quella di Carafa, sino alla breve ascesa al pontificato. Poi fu la volta di Paolo IV, che con la costituzione Cum ex apostolatus officio sbarrò per sempre l'accesso al papato ai candidati non graditi al Sant'Uffizio. Morone subì l'onta della prigionia, ma dopo la morte di Carafa fu rapidamente riabilitato dal papa milanese e filo-imperiale Pio IV, che per le sue scelte avrebbe meritato insinuanti accuse di eresia. L'odissea del processo dell'Inquisizione spagnola contro il primate Bartolomé de Carranza (1559-1576) e la progressiva distanza tra le corti di Madrid e di Vienna avrebbe sottratto a Morone l'appoggio di Filippo II; tuttavia la sintonia con papa Medici e quella con la linea imperiale e anti-inquisitoriale che stentò a prevalere a Roma fecero sì che negli anni di Pio IV Morone potesse mettere a frutto tutta la propria abilità diplomatica. Fu lui infatti a „salvare“ il concilio dalle aspre divisioni in materia di origine divina dell'obbligo di residenza dei vescovi e a condurre in porto quella „Illiade“ del secolo XVI a tutto vantaggio della Sede apostolica. Legato a Carlo Borromeo, il Morone degli ultimi anni, il presidente della fase finale del Tridentino, è uno stimato uomo di Curia che però nel conclave del 1566 avrebbe mancato ancora una volta l'occasione di diventare papa. Eletto Ghislieri, com'era facile prevedere le indagini contro di lui continuarono (ma un processo non poté concludersi per ragioni di opportunità, pp. 720 sg.), mentre nella gerarchia ecclesiastica e nella Penisola italiana ciò che restava del dissenso ereticale veniva liquidato con durezza inaudita. Tornato a Modena, il cardinale cooperò alle azioni inquisitoriali ma non senza fare ricorso a qualche abiura in segreto (p. 740): segno che la linea del Sant'Uffizio non fu mai la sua. E quando quella stagione della Chiesa si chiuse con l'elezione del papa giurista Ugo Boncompagni, Morone poté terminare gli anni travagliati della sua vita circondato dalla stima curiale nonostante la persistenza di ombre del passato (per un paradosso, venne sepolto alla Minerva, non lontano dalla statua che effigia l'arcinemico Carafa). Non vi è spazio per entrare nei dettagli delle ultime parti del volume, ricche di spunti e di piste innovative. Basterà accennare al fatto che si conclude con un'indagine minuziosa sui conti del cardinale, sul momento in cui presiedette alla gestione del santuario più importante del tempo (la Santa Casa di Loreto) e sul suo ruolo come protettore della nazione inglese e di diversi Ordini (gli amati padri gesuiti, ma anche i frati domenicani e i monaci cister- 
censi e benedettini cassinesi, già sospetti di pelagianesimo). Infine, pagine complesse sono dedicate all'abilità con cui nel 1576 Morone seppe dare una soluzione istituzionale agli aspri conflitti che dividevano il patriziato genovese (cap. XXV) e alle ultime missioni a Vienna e in Germania nei primi anni della riconquista cattolica, quando il prelato milanese contribuì a delineare una Ostpolitik pontificia in direzione della Polonia di Stefano Báthory e persino della Moscovia di Ivan IV.

Nella sua insistenza sui conflitti in seno alla Chiesa negli anni centrali del XVI secolo il libro ha espliciti obiettivi polemici: da un lato la stantia riproposizione della categoria di „riforma cattolica“, e dall’altro la nuova corrente di indagini sul „rinnovamento cattolico“ che ha un punto di riferimento in John O’Malley e nella storiografia anglosassone, che stenterebbe a impiegare un termine tanto pregno di significati come „controriforma“. Da una tale prospettiva, secondo gli autori, si finisce per edulcorare la violenza con cui fu liquidata ogni visione alternativa della fede in Cristo e si occultano le cicatrici che la Chiesa avrebbe portato su di sé fino a tempi assai recenti. Si tratta di una presa di posizione coraggiosa e in larga parte rispondente alla verità (almeno se si guarda all'Europa). Tuttavia, nonostante i risvolti politici filo-imperiali che ebbe, la parabola degli „spirituali“ genera una certa amarezza per il fatto di apparire quasi alla stregua di un'inconcludente „religioseria“ (Carlo Dionisotti) destinata a perdere davanti alla linea ben più chiara del Sant’Uffizio, senza contare le prebende che il circolo di nobili e colti chierici attratti dal linguaggio valdesiano avrebbero perduto se si fossero posti in aperta rotta di collisione con il papato, vero dominus beneficiorum della Penisola italiana. Inoltre il titolo del libro stona un poco con quanto gli autori ci raccontano: al di là del fatto che usare la definizione di eretico significa mettersi sul sentiero poliziesco degli inquisitori, Morone fu sedotto dalla prospettiva degli „spirituali“ in una precisa congiuntura e per un breve tratto della sua carriera di alto prelato conclusasi nel segno di Roma. Certo, la sua proposta non fu affatto quella dell'intransigente Carafa; ma se l'uomo non fu privo di percezione della „realtà effettuale“ (pp. XVI, XVIII, 45, 203), allora non occorre sovrastimarne il dissenso né monumentalizzarne la figura dando così ragione ai suoi persecutori. 